\title{
Green Synthesis of Ag Nanoparticles by Celery Leaf Extract Supported on Magnetic Carbon-Based Biochar Substrate, as a Green Catalyst for Reduction Reactions
}

\author{
Sahar Taheri \\ Alzahra University \\ Majid M Heravi ( $\square$ mmheravi@alzahra.ac.ir) \\ Alzahra University \\ Pourya Mohammadi \\ Alzahra University
}

\section{Research Article}

Keywords: Green synthesis, Ag nanoparticles, biochar, Reduction reaction, Magnetic nanocatalyst

Posted Date: October 14th, 2021

DOl: https://doi.org/10.21203/rs.3.rs-941842/v1

License: (c) (1) This work is licensed under a Creative Commons Attribution 4.0 International License.

Read Full License 


\section{Abstract}

Green synthesis of a noble metal such as Ag nanoparticles is an enormously developed research area. In this study, biochar $/ \mathrm{Fe}_{3} \mathrm{O}_{4}-\mathrm{Ag}$ magnetic nanocatalyst was produced via a green path by using Celery stalk as a carbon-based substrate, and Celery leaf extract as reducing and stabilizing agents to construct Ag nanoparticles. The synthesized nanocatalyst was determined using various techniques, such as UV-Vis, FT-IR spectroscopy, XRD (X-ray diffraction), SEM/EDX spectroscopy (scanning electron microscopy/energy-dispersive X-ray), TEM (transmission electron microscopy), and VSM (vibrating sample magnetometer). To survey the catalytic action of the biochar $/ \mathrm{Fe}_{3} \mathrm{O}_{4}$-Ag nanocatalyst, it was used in the reduction reaction of disparate nitroaromatics, aldehydes, and ketones. This catalyst has demonstrated premier characteristics in terms of the amount, reusability, recoverability, activity, and structural integrity of the catalyst during the reaction. In addition, biochar $/ \mathrm{Fe}_{3} \mathrm{O}_{4}$ - $\mathrm{Ag}$ could be detached magnetically and recycled multiple times without significantly reducing its catalytic performance.

\section{Introduction}

Biochar is a stable and carbon-rich solid that is produced by the pyrolysis of diverse biomasses, such as wood, manure, or leaves, without the presence of oxygen (or only a very small amount) [1-3]. Additionally, biochar can be acquired from raw feedstock such as plant-based raw materials [4]. Biochar has supreme features such as rich carbon content, wide specific surface area, stable and porous structure, high ion exchange capacity, numerous functional groups, easy access, and low price of raw materials [5-7]. Because of its substantial characteristics, such as large surface area, abundant active functional groups, porosity, and pore volume, it has been used for a variety of applications, including environmental remediation, energy storage, water, and wastewater treatment, catalyst support, etc. [8, 9]. To improve the physicochemical properties of biochar, different processes such as modification with acids, alkalis, oxidizing agents, and various activation methods, including amination, hydrothermal synthesis and magnetization have been employed [10-12].

Catalysts are very important for human society since more than $90 \%$ of all chemical processes need a catalyst for the targeted generation of various products [13]. In recent years, the benefit of noble metal nanoparticles as heterogeneous catalysts has gained much consideration because of their unparalleled virtues such as large surface area and high chemical activity [14-16]. Silver NPs, as substantial noble metal NPs, have been most extensively utilized and studied owing to their stability, high conductivity, antibacterial properties, specific virtues of size and shape, and particularly their superb catalytic activity [17-19]. Multiple approaches exist for the synthesis of silver NPs [20]. For instance, silver ions are reduced by chemical [21, 22], photochemical [23], radiation [24], electrochemical [25, 26], Langmuir Blodgett [27, 28], and biological techniques [29]. The naturally occurring substances for biological synthesis consist of plant extracts, plants, and microorganisms [30]. In addition, the green synthesis process is a convenient way to synthesize NPs because it is not only a one-step and eco-friendly method of synthesis but also reduces the consumption or production of hazardous materials to human health and the environment [31-34]. 
Recently, the utilization of magnetic nanoparticles (MNPs) as a beneficial material has increased significantly owing to their specific virtues, such as high specific surface area, superparamagnetic properties, small size, nontoxicity, and large surface-to-volume ratio [35, 36]. The accumulation of nanoparticles during the catalytic reaction is one of their impediments [37]. Segregation of nanoparticles from the reaction medium using ordinary methods such as filtration and centrifugation is tough.

Therefore, magnetic nanoparticles allow easygoing dissociation from the reaction mixture via an external magnet without the need for further processes that not only omit the necessity of cumbersome centrifugation and filtration procedures but also diminish energy expenditure, and catalyst wastage and preserve time in attainment catalyst recovery [38-41].

Reduction reactions are one of the most substantial and beneficial chemical reactions in the synthesis of organic compounds [42]. Aromatic nitro compounds are widely utilized in different processes. When these compounds are released into the environment, they cause severe injuries to humans, plants, animals, and the whole biological system. From this point of view, the constitution of amines through the reduction of nitro compounds represents a fundamental evolution in organic chemistry [43, 44]. Amines, especially aniline and its derivatives, which are indispensable for the construction of pesticides, pigments, polymers, dye intermediates, and pharmaceutics, play a momentous role in the organic chemical industry [45-47]. On the other hand, the reduction of aldehydes and ketones is significant due to the far-reaching utilization of alcohol in pharmaceuticals, agrochemicals, and the preparation of solvents [48]. From petroleum products, we will not obtain a vast range of alcohols, but performing aldehyde, and ketone reduction reactions will result in the provision of various alcohols [49]. The tense issue in the synthesis of amines and alcohols is to select the most efficient heterogeneous catalyst to acquire the best catalytic activity, reusability, and persistence. For this purpose many studies have been accomplished on the reduction of nitro, aldehyde, and ketone compounds in the attendance of diverse heterogeneous catalysts [50-54]. For instance, various solid supports such as $\mathrm{TiO}_{2}$ [55], carbon-based materials (biochar, graphene) [56], and $\mathrm{Fe}_{3} \mathrm{O}_{4}$ have been used to stabilize metal nanoparticles and fabricate heterogeneous nanocatalysts $[57,58]$.

Herein, we have reported a green process to provide a novel nanoscale silver particle catalyst supported on a magnetic carbon-based biochar substrate. For the synthesis of the biochar/ $/ \mathrm{Fe}_{3} \mathrm{O}_{4}-\mathrm{Ag}$ nanocatalyst, initially, a carbon-based biochar substrate was constructed using a celery plant, and then a biochar $/ \mathrm{Fe}_{3} \mathrm{O}_{4}$ nanocomposite was prepared in the presence of magnetic nanoparticles. Finally, silver nanoparticles were synthesized utilizing celery leaf extract and immobilized on a magnetic biochar/ $/ \mathrm{Fe}_{3} \mathrm{O}_{4}$ substrate. Therefore, we synthesized a biochar/ $/ \mathrm{Fe}_{3} \mathrm{O}_{4}$-Ag nanocatalyst and exploited it in the reduction of nitroaromatic, aldehyde, and ketone compounds using $\mathrm{NaBH}_{4}$ as the reducing agent and $\mathrm{H}_{2} \mathrm{O}$ as the green solvent. In general, the green synthesized nanocatalyst was manufactured using green precursors, solvents, and methods.

\section{Experimental}




\section{Materials}

Fresh Celery, ferric chloride hexahydrate $\left(\mathrm{FeCl}_{3} \cdot 6 \mathrm{H}_{2} \mathrm{O}, 97 \%\right)$, ferrous chloride tetrahydrate $\left(\mathrm{FeCl}_{2} \cdot 4 \mathrm{H}_{2} \mathrm{O}\right.$, $98 \%)$, ammonium hydroxide solution $\left(\mathrm{NH}_{4} \mathrm{OH}, 25 \%\right)$, and silver nitrate $\left(\mathrm{AgNO}_{3}, 99 \%\right)$ were procured from Sigma-Aldrich. Aldehyde and ketone derivatives were purchased from Sigma and Merck. Celery leaves have been purchased from a local market in Tehran, Iran and this study complies with relevant institutional, national, and international guidelines and legislation.

\section{Synthesis of Biochar $/ \mathrm{Fe}_{3} \mathrm{O}_{4}-\mathrm{Ag}$ nanocatalyst}

\section{Synthesis of Biochar}

To prepare the biochar carbon substrate initially, $250 \mathrm{~g}$ of celery stalk was rinsed and desiccated at $60^{\circ} \mathrm{C}$ for $24 \mathrm{~h}$ and then powdered. The green powder $(4 \mathrm{~g})$ with distilled water $(65 \mathrm{~mL})$ was poured into a 100 $\mathrm{mL}$ autoclave. Approximately, the autoclave was warmed up at $180 \mathrm{C}$ for $24 \mathrm{~h}$, and then chilled naturally to ambient temperature. The solid vintage was separated by centrifugation, washed and, dried. The final black powder was biochar.

\section{Synthesis of Biochar $/ \mathrm{Fe}_{3} \mathrm{O}_{4}$}

Normally, $0.5 \mathrm{~g}$ of biochar is interspersed into $120 \mathrm{~mL}$ of distilled $\mathrm{H}_{2} \mathrm{O}$. Then, $0.5 \mathrm{~g}$ of $\mathrm{FeCl}_{2} .4 \mathrm{H}_{2} \mathrm{O}$ and 1.37 $\mathrm{g}$ of $\mathrm{FeCl}_{3} \cdot 6 \mathrm{H}_{2} \mathrm{O}$ were surcharged and warmed at $35^{\circ} \mathrm{C}$ for $3 \mathrm{~h}$. Subsequently, the temperature was up to $60{ }^{\circ} \mathrm{C}$, and $10 \mathrm{~mL}$ of $\mathrm{NH}_{4} \mathrm{OH}$ was augmented dropwise to the above blend. Then, the mixture was stirred for an additional $60 \mathrm{~min}$. After cooling to room temperature, the product was segregated using an external magnet, was eluted multiple times with distilled water $\left(\mathrm{H}_{2} \mathrm{O}\right)$, and dried at ambient temperature.

\section{Preparation of leaf extract}

One hundred grams of green celery leaves was cut and thoroughly rinsed many times with distilled water $\left(\mathrm{H}_{2} \mathrm{O}\right)$ to eliminate mist particles. Afterward, the green leaves $(50 \mathrm{~g})$ were extracted by using $300 \mathrm{~mL}$ of distilled water at $100^{\circ} \mathrm{C}$ for $6 \mathrm{~h}$, after which, they were permitted to become cold on their own. Finally, the celery leaf extract solution was filtered and dried at $55^{\circ} \mathrm{C}$. The procurement of leaf extract is shown in Fig.S1.

\section{Green synthesis of Ag NPs}

In a usual reaction process, $2 \mathrm{~mL}$ of the leaf extract of $0.25 \% \mathrm{M}$ was added dropwise to $5 \mathrm{~mL}$ of $1.5 \mathrm{mM}$ aqueous $\mathrm{AgNO}_{3}$ solution and stirred at $55^{\circ} \mathrm{C}$. The $\mathrm{Ag} \mathrm{NPs}$ were made from the reduction of silver ions over approximately $3 \mathrm{~h}$. By UV-visible (UV-Vis) spectroscopy, the reaction was controlled. The color change from light green to brown confirms the reduction of $\mathrm{Ag}^{+}$to $\mathrm{Ag}^{0}$.

\section{Green synthesis of Biochar $/ \mathrm{Fe}_{3} \mathrm{O}_{4}-\mathrm{Ag}$}


To procure the nanocatalyst, biochar $/ \mathrm{Fe}_{3} \mathrm{O}_{4}(0.15 \mathrm{~g})$ was appended to $20 \mathrm{~mL}$ of distilled water $\left(\mathrm{H}_{2} \mathrm{O}\right)$ and stirred for $30 \mathrm{~min}$. Afterward, $50 \mathrm{~mL}$ of $\mathrm{AgNO}_{3}(2 \mathrm{mM})$ solution was appended into the blend and stirred at room temperature for $5 \mathrm{~h}$. Thereafter, the temperature of the reaction was up to $65^{\circ} \mathrm{C}$, and it was stirred for another $30 \mathrm{~min}$. Afterward, $10 \mathrm{~mL}$ of aqueous extract $(0.25 \% \mathrm{M})$ was appended into this blend and stirred for $3 \mathrm{~h}$ at $65^{\circ} \mathrm{C}$. The constructed product was detached by an external magnet, eluted with distilled water $\left(\mathrm{H}_{2} \mathrm{O}\right)$ a couple of times, and desiccated at ambient temperature. The process of biochar/ $/ \mathrm{Fe}_{3} \mathrm{O}_{4}-\mathrm{Ag}$ nanocatalyst synthesis is shown in Fig. S2.

\section{Reduction of Nitroaromatic compounds catalyzed by Biochar $/ \mathrm{Fe}_{3} \mathrm{O}_{4}-\mathrm{Ag}$}

Catalytic reduction reactions of nitro compounds were performed in an aqueous solution at $50{ }^{\circ} \mathrm{C}$ in the presence of $\mathrm{NaBH}_{4}$ as the reducing agent. In a typical way, nitroaromatic compounds $(0.5 \mathrm{mmol})$ and $\mathrm{H}_{2} \mathrm{O}(3.0 \mathrm{~mL})$ were mixed into a $10 \mathrm{~mL}$ round-bottom flask and stirred at the desired temperature. Then biochar $/ \mathrm{Fe}_{3} \mathrm{O}_{4}$-Ag nanocatalyst $(10 \mathrm{mg})$ and $\mathrm{NaBH}_{4}(3 \mathrm{mmol})$ were added, and the final mixture was stirred for a suitable time. The progression of the reaction was controlled by applying TLC (normal hexane-ethyl acetate as solvent). After the finishing of the reduction reaction, the biochar $/ \mathrm{Fe}_{3} \mathrm{O}_{4}-\mathrm{Ag}$ nanocatalyst was segregated by an outer magnet, rinsed with $\mathrm{H}_{2} \mathrm{O}$ and ethanol, and dried to be applied for the next cycle. Finally, to provide pure products, the obtained products were recrystallized from ethanol.

\section{Reduction of aldehyde and ketone compounds catalyzed by biochar $/ \mathrm{Fe}_{3} \mathrm{O}_{4}-\mathrm{Ag}$}

In a catalytic process, the reduction reactions of aldehyde and ketone compounds were performed. In a usual way, a mixture of $0.5 \mathrm{mmol}$ aldehyde and ketone compounds, and $2 \mathrm{~mL}$ of water as a solvent was added into a round bottom flask and stirred for $10 \mathrm{~min}$ at ambient temperature. Afterward, $5 \mathrm{mg}$ of biochar $/ \mathrm{Fe}_{3} \mathrm{O}_{4}$-Ag catalyst and $\mathrm{NaBH}_{4}(3 \mathrm{mmol})$ were subjoined into the above mixture, and the whole combination was stirred for an adequate time. The reaction was monitored by TLC (normal hexane-ethyl acetate as the solvent, 2:8). After termination of the reaction, the nanocatalyst was detached by an outer magnet, and rinsed with ethanol and $\mathrm{H}_{2} \mathrm{O}$, dried to be used in the next cycle. Finally, the product was extracted and purified.

\section{Results}

\section{FT-IR spectroscopy}

To study the structure of the presented nanocatalyst in more detail and characterize functional groups, the FT-IR spectra of the nanocatalyst fabrication steps of (a) biochar (b) biochar- $\mathrm{Fe}_{3} \mathrm{O}_{4}$, and (c) Biochar $/ \mathrm{Fe}_{3} \mathrm{O}_{4}-\mathrm{Ag}$ were examined, and the results are shown in Fig.S3. The peaks present at 3300-3500 $\mathrm{cm}^{-1}$ are linked to the $\mathrm{OH}$ stretching vibration mode in Fig.S3a. The uptake peaks at $2852 \mathrm{~cm}^{-1}$ and 2923 $\mathrm{cm}^{-1}$ were caused by the stretching vibration of the $\mathrm{C}-\mathrm{H}$ bond. The bonds observed at $1733 \mathrm{~cm}^{-1}, 1650$ 
$\mathrm{cm}^{-1}$, and $1061 \mathrm{~cm}^{-1}$ were devoted to the presence of stretching vibrations of the carbonyl group (C=0), $\mathrm{C}=\mathrm{C}$, and $\mathrm{C}-\mathrm{O}$ bonds, respectively. In addition, the broad peak at $700-800 \mathrm{~cm}^{-1}$ corresponded to the out-ofplane $\mathrm{C}-\mathrm{H}$ band. In Fig.S3b, the sharp absorption bond at $580 \mathrm{~cm}^{-1}$ was ascribed to the tetrahedral structure of the $\mathrm{Fe}-\mathrm{O}$ bond. Fig.S3c shows that by adding silver nanoparticles to the surface of the biochar- $\mathrm{Fe}_{3} \mathrm{O}_{4}$ substrate, there was no considerable change in the spectrum. Therefore, it can be concluded that the biochar- $\mathrm{Fe}_{3} \mathrm{O}_{4}$ substrate was stable during the synthesis of silver $(\mathrm{Ag})$ nanoparticles.

\section{X-ray diffraction (XRD)}

In Fig.S4, the X-ray diffraction patterns of the silver (Ag) nanoparticles synthesized from the extract are shown. The peaks were detected at $2 \theta$ values of $77.31^{\circ}, 64.5^{\circ}, 57.47^{\circ}, 54.98^{\circ}, 46.4^{\circ}, 44.26^{\circ}, 38.13^{\circ}$, $32.22^{\circ}$, and $27.92^{\circ}$ which are related to the (311), (220), (241), (142), (231), (200), (111), (122) and (210) silver crystalline planes, with face-centered cubic structures (JCPDS, file No. 04-0783). The silver nanoparticles synthesized by the extract, have a crystal nature that is observable according to the XRD results. The average crystallite size of silver $(\mathrm{Ag})$ nanoparticles was measured by applying DebyeScherer's equation. The size of the achieved Ag nanoparticles was calculated at $15.2 \mathrm{~nm}$ from the breadth of the plane (122) reflection.

The XRD analysis of the biochar/ $\mathrm{Fe}_{3} \mathrm{O}_{4}$-Ag nanocomposite is exhibited in Fig.S5. In this pattern, a characteristic peak at $22.8^{\circ}$ was observed, which was related to the reflection of the (002) crystal plane of carbon-based biochar substrate. The diffraction peaks at $2 \nabla=62.84^{\circ}, 57.22^{\circ}, 53.68^{\circ}, 43.26^{\circ}, 35.59^{\circ}$, and $30.22^{\circ}$ were ascribed to the reflection planes of the (440), (511), (422), (400), (311) and (220) for $\mathrm{Fe}_{3} \mathrm{O}_{4}$, respectively. These patterns confirmed the face-centered cubic structure of $\mathrm{Fe}_{3} \mathrm{O}_{4}$ nanoparticles (JCPDS card no. 19-0629). Additionally, several Bragg reflection peaks were observed at $32.09^{\circ}, 38.18^{\circ}, 44.38^{\circ}$, $64.57^{\circ}$, and $77.56^{\circ}$, which can be ascribed to the reflections of the (122), (111), (200), (220), and (311) crystalline phases of the fcc-structured Ag nanoparticles, respectively (JCPDS card no. 65-2871). Using Debye-Scherer's equation, the crystallite size of nanoparticles was calculated to be $13.4 \mathrm{~nm}$ for $\mathrm{Fe}_{3} \mathrm{O}_{4}$ from the width of the plane (311) and $19.8 \mathrm{~nm}$ for Ag from the width of the plane (111).

\section{UV-Vis Analysis of silver (Ag) NPs}

UV-Vis analysis of leaf extract, silver nitrate $\left(\mathrm{AgNO}_{3}\right)$, and synthesized silver NP spectra are shown in Fig. 1. In this process, Ag NPs were prepared using Celery leaf extract. Reducing $\mathrm{Ag}^{+}$into $\mathrm{Ag}^{0}$ was upheld by changing the color of the reaction mixture from light green to brown. As shown in Figure 1, the leaf extract and $\mathrm{AgNO}_{3}$ solution did not show any absorbance peak in the range of $400-800 \mathrm{~nm}$, but by appending the leaf extract into the $\mathrm{AgNO}_{3}$ solution, good absorbance was observed at $440 \mathrm{~nm}$, which was relevant to the SPR (surface plasmon resonance) of silver (Ag) NPs.

\section{FESEM and TEM analysis}


The FESEM (field emission scanning electron microscopy) images of the biochar $/ \mathrm{Fe}_{3} \mathrm{O}_{4}-\mathrm{Ag}$ nanocatalyst in the range of $500 \mathrm{~nm}-2 \mu \mathrm{m}$ are shown in Figure $2(a-b)$, which illustrates that the nanocatalyst consists of $\mathrm{Fe}_{3} \mathrm{O}_{4}$ and $\mathrm{Ag}$ spherical nanoparticles. It was also observed that the approximate size of the nanoparticles was 45-50 nm. FESEM images of biochar $/ \mathrm{Fe}_{3} \mathrm{O}_{4}-\mathrm{Ag}$ show that the surfaces of biochar are well decorated with $\mathrm{Ag}$ and $\mathrm{Fe}_{3} \mathrm{O}_{4}$ nanoparticles. Figure 2 (c-d) represents the TEM images of the obtained nanocomposite. The TEM images indicate the distribution of $\mathrm{Fe}_{3} \mathrm{O}_{4}$ and $\mathrm{Ag}$ NPs with a size range of $40-50 \mathrm{~nm}$ on the biochar substrate. The size and morphology of Ag nanoparticles depended on the green extract used for synthesis.

\section{Energy dispersive spectrometry (EDS)-Mapping}

To further investigate the structure of the biochar/ $\mathrm{Fe}_{3} \mathrm{O}_{4}$ - $\mathrm{Ag}$ nanocomposite, EDS mapping analysis was used to detect the distribution of the elements on the surface of the nanocomposite. Figure 3 represents the EDS mapping images of this nanocomposite. In light of the outcomes, the presence of the $\mathrm{C}, \mathrm{O}, \mathrm{Fe}$, and $\mathrm{Ag}$ elements in the structure of the biochar/ $/ \mathrm{Fe}_{3} \mathrm{O}_{4}$-Ag nanocatalyst was confirmed, which confirms for the consolidation of $\mathrm{Fe}_{3} \mathrm{O}_{4}$ and $\mathrm{Ag}$ nanoparticles on the carbon-based biochar substrate.

\section{Vibration sampling magnetometer (VSM) analysis}

The appraisal of the magnetic properties of the biochar/ $/ \mathrm{Fe}_{3} \mathrm{O}_{4}-\mathrm{Ag}$ nanocomposite was carried out utilization the VSM technique. The VSM chart of the biochar $/ \mathrm{Fe}_{3} \mathrm{O}_{4}$-Ag nanocomposite is shown in Figure 4. According to the curve obtained from the VSM, biochar/ $/ \mathrm{Fe}_{3} \mathrm{O}_{4}$-Ag has magnetic properties, and its saturation magnetization value was $29.4 \mathrm{emu} / \mathrm{g}$. Additionally, due to the absence of a hysteresis loop, this nanocomposite has superparamagnetic properties. This magnetic behavior of the prepared nanocatalyst, causes the particles to accumulate rapidly in the attendance of an external magnet, and the particles are easily dispersed as soon as the external magnet is removed.

\section{Catalytic performance}

\section{Nitroaromatic compounds reduction reaction}

The reduction of 4-nitroaniline $(0.5 \mathrm{mmol})$ was considered the model reaction to optimize the reduction reaction conditions of nitroaromatics. The quantity of biochar/ $/ \mathrm{Fe}_{3} \mathrm{O}_{4}$ - $\mathrm{Ag}$ nanocatalyst, temperature, and type of solvent were changed and evaluated to achieve the optimized value as shown in Table S1.

To optimize the reaction conditions, first, different amounts of catalyst were examined. The results showed that, in the absence of the catalyst, the reduction reaction did not occur (Table S1, Entry 1). Therefore, the presence of biochar $/ \mathrm{Fe}_{3} \mathrm{O}_{4}-\mathrm{Ag}$ nanocatalysts is a vital factor for the reduction reaction. As seen, $10 \mathrm{mg}$ of catalyst was considered a reasonable and optimal value (Table S1, Entry 4). Additionally, based on the results, it was found that by increasing the amount of nanocatalyst, the reaction time was decreased and the yield was increased (Table S1, Entries 2-5). 
After determining the optimal value of the nanocatalyst, the efficacy of temperature on the progress of the reduction reaction was surveyed. Based on the outcomes, it was observed that increasing the temperature led to higher performance and yield of the reaction as well as decreasing the reaction time (Table S1, Entries 6,7$)$. Therefore, due to less energy consumption, $50^{\circ} \mathrm{C}$ was selected as the appropriate and optimal temperature for this reaction (Table S1, Entry 4).

Finally, to investigate the effect of the solvent, the model reaction was performed in the presence of different solvents (Table S1, Entries 8-13). Following the outcomes, $\mathrm{H}_{2} \mathrm{O}$, as a green, cheap and stable solvent, indicated the best performance with a $98 \%$ yield (Table S1, Entry 4). These results illustrate that biochar $/ \mathrm{Fe}_{3} \mathrm{O}_{4}$-Ag has good catalytic efficiency for the reduction of nitroaromatic compounds by utilization of $\mathrm{NaBH}_{4}$ as the reducing agent in water.

Following the results of the optimization experiments, the optimal conditions for the reduction reaction of nitroaromatic compounds were $10 \mathrm{mg}$ of the biochar $/ \mathrm{Fe}_{3} \mathrm{O}_{4}-\mathrm{Ag}$ synthesized nanocatalyst in water at 50 ${ }^{\circ} \mathrm{C}$. After gaining the optimized conditions, the reduction reactions of different nitroaromatic compounds under these conditions are investigated and the results were illustrated in Table S2. The first compound, 4-nitroaniline was reduced at a yield of $98 \%$ in 60 min (Table S2, Entry 1). Substituted nitroaromatic compounds such as amine, acid, and hydroxyl-nitrobenzenes were also reduced with high reaction performance and a yield of more than $95 \%$ in the reaction time range of 40-80 min (Table S2, Entries 2-8).

\section{Reusability of the biochar $/ \mathrm{Fe}_{3} \mathrm{O}_{4}-\mathrm{Ag}$ nanocatalyst for the nitroaromatic reduction reaction}

Recyclability is an important factor to evaluate a catalyst. Therefore, recycling experiments were accomplished to appraise the stability and activity of the catalyst. Under the optimized conditions, the biochar/ $/ \mathrm{Fe}_{3} \mathrm{O}_{4}-\mathrm{Ag}$ nanocatalyst was separated by an external magnet after the nitroaromatic compound reduction reaction was completed, and then it was washed, dried, and used for subsequent cycles. The recovered catalyst was reused up to 5 times without substantial reduction in catalytic activity, and the results are demonstrated in Figure 5. These results demonstrated that the biochar/ $/ \mathrm{Fe}_{3} \mathrm{O}_{4}$ - $\mathrm{Ag}$ catalyst has privileged properties such as good catalytic performance, cost-effectiveness, facile and green synthesis, good stability, and recyclability, which made it an adequate catalyst for the reduction of nitroaromatic compounds.

\section{Aldehyde and ketone compounds reduction reaction}

To optimize the reduction reaction conditions of aldehydes and ketones, such as the amount of biochar/ $/ \mathrm{Fe}_{3} \mathrm{O}_{4}$-Ag nanocatalyst, temperature, type of solvent, and the amount of $\mathrm{NaBH}_{4}$ as a reducing agent, the reduction of benzaldehyde $(0.5 \mathrm{mmol})$ as a model reaction was examined, and it is demonstrated in Table 2.

First, the effect of the biochar/ $/ \mathrm{Fe}_{3} \mathrm{O}_{4}$-Ag amount was determined by keeping the other reaction conditions firm. In the absence of the catalyst, the reduction reaction time was long (Table 1, Entry 1 ). Additionally, 
according to the results, it was found that by adding the amount of biochar/ $\mathrm{Fe}_{3} \mathrm{O}_{4}$-Ag nanocatalyst, the reaction time decreased and the yield increased (Table 1, Entries 2-4). Therefore, $5 \mathrm{mg}$ of biochar $/ \mathrm{Fe}_{3} \mathrm{O}_{4^{-}}$ Ag was selected as the optimal value, because of the short reaction time and high yield (Table 1, Entry 4).

After opting for the appropriate amount of catalyst, the efficacy of temperature on the reaction progress was determined. Depending on the results (Table 1, Entry 4), $25^{\circ} \mathrm{C}$ was determined to be the optimal temperature for this reaction, because this temperature complies with the laws of green chemistry. Additionally, it was observed that increasing the temperature led to decrease in the reaction time (Table 1, Entry 5).

After determining the appropriate temperature, the next step is to peruse the effect of the solvent on the attendance of the biochar/ $\mathrm{Fe}_{3} \mathrm{O}_{4}$-Ag catalyst in the reaction progress. As a function of the outcomes, $\mathrm{H}_{2} \mathrm{O}$ with a yield of $98 \%$ (Table 1, Entry 4 ) and THF with a yield of $96 \%$ (Table 1, Entry 9) were suitable solvents for this reaction, but $\mathrm{H}_{2} \mathrm{O}$ was selected as the optimal solvent due to its green, inexpensive and high yield. Additionally, other solvents such as ethanol, acetonitrile, and DMF had a yield between $60-87 \%$ (Table 1 , Entries 6, 8, 11).

Finally, $\mathrm{NaBH}_{4}$ was used as a reducing agent, and its different concentrations were investigated to assess the optimum value while maintaining that the other parameters were constant. With the enhancement of the concentration of $\mathrm{NaBH}_{4}$, the yield was enhanced (Table 1, Entries 13, 14). Therefore, the concentration of $1 \mathrm{mmol} \mathrm{NaBH}_{4}$ was determined as the optimized value (Table 1, Entry 4). These results elucidate that biochar $/ \mathrm{Fe}_{3} \mathrm{O}_{4}$-Ag is an outstanding catalyst for the reduction of aldehyde and ketone compounds in the presence of $\mathrm{NaBH}_{4}$ as the reducing agent in water.

As a first example, benzaldehyde was reduced at a yield of $98 \%$ in 3 min (Table 2, Entry 1). Substituted aldehyde and ketone compounds were also reduced with great reaction performance, the yield was more than $85 \%$ in the reaction time range from 3 to 10 min for aldehydes, and the yield was more than $30 \%$ in the reaction time range from 6 to $60 \mathrm{~min}$ for ketones.

\section{Reusability of the biochar $/ \mathrm{Fe}_{3} \mathrm{O}_{4}-\mathrm{Ag}$ nanocatalyst for aldehyde and ketone reduction reactions}

Catalyst recovery and reusability are fundamental factors in the nomination of the efficiency and performance of the catalyst. In this regard, the recoverability of the biochar $/ \mathrm{Fe}_{3} \mathrm{O}_{4}-\mathrm{Ag}$ nanocatalyst was investigated in the model reaction of the reduction of aldehydes and ketones under the optimal conditions. The results of the performance testing demonstrated, that the synthesized nanocatalyst could be applied in at least 10 successive runs without a substantial reduction in catalytic performance, which affirmed the heterogeneous nature of the catalyst. The results are shown in Figure 6. Based on the outcomes, good catalytic performance, excellent stability, and recoverability, biochar/ $/ \mathrm{e}_{3} \mathrm{O}_{4}-\mathrm{Ag}$ is an appropriate catalyst for the reduction of aldehydes and ketones.

\section{Conclusion}


This study was centralized on the green synthesis of biochar as a carbon-based substrate and Ag nanoparticles using leaf extract of Celery. In general, a fast and ecofriendly synthesis process for silver nanoparticles in the presence of a green precursor and solvent has been illustrated. The structural, morphological, and optical properties of the Ag NPs, were determined by diverse techniques. The prepared biochar $/ \mathrm{Fe}_{3} \mathrm{O}_{4}-\mathrm{Ag}$ nanocatalyst exhibited excellent catalytic efficiency for the reduction of nitroaromatic compounds, aldehydes, ketones in the presence of $\mathrm{NaBH}_{4}$ as a reducing agent, and $\mathrm{H}_{2} \mathrm{O}$ as green a solvent, and possessed appropriate reusability. The advantages of this heterogeneous catalyst include green conditions such as low reaction temperature, green solvent, short reaction time, easy separation, low cost, and ecofriendly. The biochar $/ \mathrm{Fe}_{3} \mathrm{O}_{4}$ - $\mathrm{Ag}$ nanocatalyst could be segregated by the utilization of an external magnet and reused five to ten times without appreciable loss of its catalytic performance.

\section{References}

[1] Y. Li, B. Xing, X. Wang, K. Wang, L. Zhu, and S. Wang, "Nitrogen-Doped Hierarchical Porous Biochar Derived from Corn Stalks for Phenol-Enhanced Adsorption," Energy and Fuels, vol. 33, no. 12, pp. 1245912468, Dec. 2019, doi: 10.1021/acs.energyfuels.9b02924.

[2] F. Guo et al., "A simple method for the synthesis of biochar nanodots using hydrothermal reactor," MethodsX, vol. 7, p. 101022, 2020, doi: 10.1016/j.mex.2020.101022.

[3] Z. Ma et al., "Evolution of the chemical composition, functional group, pore structure and crystallographic structure of bio-char from palm kernel shell pyrolysis under different temperatures," $J$. Anal. Appl. Pyrolysis, vol. 127, pp. 350-359, Sep. 2017, doi: 10.1016/j.jaap.2017.07.015.

[4] A. Enders, K. Hanley, T. Whitman, S. Joseph, and J. Lehmann, "Characterization of biochars to evaluate recalcitrance and agronomic performance," Bioresour. Technol., vol. 114, pp. 644-653, 2012, doi: 10.1016/j.biortech.2012.03.022.

[5] X. Pan, Z. Gu, W. Chen, and Q. Li, "Preparation of biochar and biochar composites and their application in a Fenton-like process for wastewater decontamination: A review," Science of the Total Environment, vol. 754. p. 142104, Feb. 2021, doi: 10.1016/j.scitotenv.2020.142104.

[6] W. Wang et al., "Preparation of TiO 2 -modified Biochar and its Characteristics of Photo-catalysis Degradation for Enrofloxacin," pp. 1-12, 2020, doi: 10.1038/s41598-020-62791-5.

[7] G. K. Parshetti, S. Chowdhury, and R. Balasubramanian, "Biomass derived low-cost microporous adsorbents for efficient CO2 capture," Fuel, vol. 148, pp. 246-254, 2015, doi: 10.1016/j.fuel.2015.01.032.

[8] J. Wang and S. Wang, "Preparation, modification and environmental application of biochar: A review," Journal of Cleaner Production, vol. 227. pp. 1002-1022, Aug. 2019, doi: 10.1016/j.jclepro.2019.04.282. 
[9] F. Lian, G. Cui, Z. Liu, L. Duo, G. Zhang, and B. Xing, "One-step synthesis of a novel N-doped microporous biochar derived from crop straws with high dye adsorption capacity," J. Environ. Manage., vol. 176, pp. 61-68, 2016, doi: 10.1016/j.jenvman.2016.03.043.

[10] J. E. Lee and Y. Park, "Applications of Modified Biochar-Based Materials for the Removal of Environment Pollutants: A Mini Review," Sustainability, vol. 12, no. 15, p. 6112, Jul. 2020, doi: $10.3390 /$ su12156112.

[11] M. B. Ahmed, J. L. Zhou, H. H. Ngo, W. Guo, and M. Chen, "Progress in the preparation and application of modified biochar for improved contaminant removal from water and wastewater," Bioresour. Technol., vol. 214, pp. 836-851, 2016, doi: 10.1016/j.biortech.2016.05.057.

[12] D. Kołodyńska, J. Bąk, M. Kozioł, and L. V. Pylychuk, "Investigations of Heavy Metal lon Sorption Using Nanocomposites of Iron-Modified Biochar," Nanoscale Res. Lett., vol. 12, no. 1, p. 433, Dec. 2017, doi: 10.1186/s11671-017-2201-y.

[13] J. Liu, J. Li, R. Ye, X. Yan, L. Wang, and P. Jian, "Versatile bifunctional nitrogen-doped porous carbon derived from biomass in catalytic reduction of 4-nitrophenol and oxidation of styrene," Chinese J. Catal., vol. 41, no. 8, pp. 1217-1229, 2020, doi: 10.1016/S1872-2067(20)63534-3.

[14] G. Marzun, A. Levish, V. Mackert, T. Kallio, S. Barcikowski, and P. Wagener, "Laser synthesis, structure and chemical properties of colloidal nickel-molybdenum nanoparticles for the substitution of noble metals in heterogeneous catalysis," J. Colloid Interface Sci., vol. 489, pp. 57-67, Mar. 2017, doi: 10.1016/j.jcis.2016.09.014.

[15] M. Govarthanan et al., "Biosynthesis and characterization of silver Nanoparticles using Panchakavya, an Indian traditional farming formulating agent," Int. J. Nanomedicine, vol. 9, no. 1, pp. 1593-1599, Mar. 2014, doi: 10.2147/IJN.S58932.

[16] Y. Zhang, D. Yang, Y. Kong, X. Wang, O. Pandoli, and G. Gao, "Synergetic Antibacterial Effects of Silver Nanoparticles @ Aloe Vera Prepared via a Green Method," Nano Biomed Eng, no. 2(4), pp. 252-257, 2010, doi: 10.5101/nbe.v2i4.p252-257.1.

[17] F. Cheng, J. W. Betts, S. M. Kelly, J. Schaller, and T. Heinze, "Synthesis and antibacterial effects of aqueous colloidal solutions of silver nanoparticles using aminocellulose as a combined reducing and capping reagent," Green Chem., vol. 15, no. 4, p. 989, 2013, doi: 10.1039/c3gc36831a.

[18] H. Veisi, S. Azizi, and P. Mohammadi, "Green synthesis of the silver nanoparticles mediated by Thymbra spicata extract and its application as a heterogeneous and recyclable nanocatalyst for catalytic reduction of a variety of dyes in water," J. Clean. Prod., vol. 170, pp. 1536-1543, Jan. 2018, doi: 10.1016/j.jclepro.2017.09.265. 
[19] M. Al-Ansari, N. Alkubaisi, K. Gopinath, V. Karthika, A. Arumugam, and M. Govindarajan, "Facile and Cost-Effective Ag Nanoparticles Fabricated by Lilium lancifolium Leaf Extract: Antibacterial and Antibiofilm Potential," J. Clust. Sci., vol. 30, no. 4, pp. 1081-1089, Jul. 2019, doi: 10.1007/s10876-01901569-w.

[20] S. Li et al., "Green synthesis of silver nanoparticles using Capsicum annuum L. Extract," Green Chem., vol. 9, no. 8, pp. 852-85, 2007, doi: 10.1039/b615357g.

[21] Y. Sun, Y. Yin, B. T. Mayers, T. Herricks, and Y. Xia, “Uniform silver nanowires synthesis by reducing AgNO3 with ethylene glycol in the presence of seeds and poly(vinyl pyrrolidone)," Chem. Mater., vol. 14, no. 11 , pp. 4736-4745, 2002, doi: $10.1021 / \mathrm{cm} 020587 \mathrm{~b}$.

[22] Y. Tan, Y. Wang, L. Jiang, and D. Zhu, "Thiosalicylic acid-functionalized silver nanoparticles synthesized in one-phase system," J. Colloid Interface Sci., vol. 249, no. 2, pp. 336-345, 2002, doi: $10.1006 /$ jcis.2001.8166.

[23] A. Callegari, D. Tonti, and M. Chergui, "Photochemically Grown Silver Nanoparticles with WavelengthControlled Size and Shape," Nano Lett., vol. 3, no. 11, pp. 1565-1568, 2003, doi: 10.1021/nl034757a.

[24] K. Mallick, M. J. Witcomb, and M. S. Scurrell, "Self-assembly of silver nanoparticles in a polymer solvent: Formation of a nanochain through nanoscale soldering," Mater. Chem. Phys., vol. 90, no. 2-3, pp. 221-224, 2005, doi: 10.1016/j.matchemphys.2004.10.030.

[25] Y. C. Liu and L. H. Lin, "New pathway for the synthesis of ultrafine silver nanoparticles from bulk silver substrates in aqueous solutions by sonoelectrochemical methods," Electrochem. commun., vol. 6, no. 11, pp. 1163-1168, 2004, doi: 10.1016/j.elecom.2004.09.010.

[26] M. A. Ebrahimzadeh, A. Naghizadeh, O. Amiri, M. Shirzadi-Ahodashti, and S. Mortazavi-Derazkola, "Green and facile synthesis of Ag nanoparticles using Crataegus pentagyna fruit extract (CP-AgNPs) for organic pollution dyes degradation and antibacterial application," Bioorg. Chem., vol. 94, p. 103425, Jan. 2020, doi: 10.1016/j.bioorg.2019.103425.

[27] L. Zhang, Y. Shen, A. Xie, S. Li, B. Jin, and Q. Zhang, "One-step synthesis of monodisperse silver nanoparticles beneath vitamin E Langmuir monolayers," J. Phys. Chem. B, vol. 110, no. 13, pp. 66156620, 2006, doi: 10.1021/jp0570216.

[28] A. Swami, P. R. Selvakannan, R. Pasricha, and M. Sastry, "One-step synthesis of ordered twodimensional assemblies of silver nanoparticles by the spontaneous reduction of silver ions by pentadecylphenol langmuir monolayers," J. Phys. Chem. B, vol. 108, no. 50, pp. 19269-19275, 2004, doi: $10.1021 /$ jp0465581.

[29] M. F. Zayed, W. H. Eisa, Y. K. Abdel-Moneam, S. M. El-kousy, and A. Atia, "Ziziphus spina-christi based bio-synthesis of Ag nanoparticles," J. Ind. Eng. Chem., vol. 23, pp. 50-56, Mar. 2015, doi: 
10.1016/j.jiec.2014.07.041.

[30] X. Zhu, K. Pathakoti, and H.-M. Hwang, "Green synthesis of titanium dioxide and zinc oxide nanoparticles and their usage for antimicrobial applications and environmental remediation," in Green Synthesis, Characterization and Applications of Nanoparticles, Elsevier, 2019, pp. 223-263.

[31] M. M. H. Khalil, E. H. Ismail, K. Z. El-Baghdady, and D. Mohamed, "Green synthesis of silver nanoparticles using olive leaf extract and its antibacterial activity," Arab. J. Chem., vol. 7, no. 6, pp. 11311139, Dec. 2014, doi: 10.1016/j.arabjc.2013.04.007.

[32] H. Bar, D. K. Bhui, G. P. Sahoo, P. Sarkar, S. P. De, and A. Misra, "Green synthesis of silver nanoparticles using latex of Jatropha curcas," Colloids Surfaces A Physicochem. Eng. Asp., vol. 339, no. 1-3, pp. 134139, 2009, doi: 10.1016/j.colsurfa.2009.02.008.

[33] L. P. Silva, T. M. Pereira, and C. C. Bonatto, "Frontiers and perspectives in the green synthesis of silver nanoparticles," in Green Synthesis, Characterization and Applications of Nanoparticles, Elsevier, 2019, pp. 137-164.

[34] A. B. Moghaddam et al., "Biosynthesis of ZnO Nanoparticles by a New Pichia kudriavzevii Yeast Strain and Evaluation of Their Antimicrobial and Antioxidant Activities," Molecules, vol. 22, no. 6, p. 872, May 2017, doi: 10.3390/molecules22060872.

[35] H. Shagholani, S. M. Ghoreishi, and M. Mousazadeh, "Improvement of interaction between PVA and chitosan via magnetite nanoparticles for drug delivery application," Int. J. Biol. Macromol., vol. 78, pp. 130-136, Jul. 2015, doi: 10.1016/j.jjbiomac.2015.02.042.

[36] J. K. Xu, F. F. Zhang, J. J. Sun, J. Sheng, F. Wang, and M. Sun, "Bio and nanomaterials based on Fe304," Molecules, vol. 19, no. 12, pp. 21506-21528, 2014, doi: 10.3390/molecules191221506.

[37] N. Esmaili, P. Mohammadi, M. Abbaszadeh, and H. Sheibani, "Green synthesis of silver nanoparticles using Eucalyptus comadulensis leaves extract and its immobilization on magnetic nanocomposite (GOFe 304 /PAA/Ag) as a recoverable catalyst for degradation of organic dyes in water," Appl. Organomet. Chem., vol. 34, no. 4, Apr. 2020, doi: 10.1002/aoc.5547.

[38] F. Paquin, J. Rivnay, A. Salleo, N. Stingelin, and C. Silva, "Multi-phase semicrystalline microstructures drive exciton dissociation in neat plastic semiconductors," J. Mater. Chem. C, vol. 3, pp. 10715-10722, 2015, doi: 10.1039/b000000x.

[39] T. Kim, X. Fu, D. Warther, and M. J. Sailor, "Size-Controlled Pd Nanoparticle Catalysts Prepared by Galvanic Displacement into a Porous Si-Iron Oxide Nanoparticle Host," ACS Nano, vol. 11, no. 3, pp. 2773-2784, Mar. 2017, doi: 10.1021/acsnano.6b07820.

[40] S. Bagheri and N. M. Julkapli, "Magnetite hybrid photocatalysis: Advance environmental remediation," Rev. Inorg. Chem., vol. 36, no. 3, pp. 135-151, 2016, doi: 10.1515/revic-2015-0014. 
[41] J. C. Colmenares et al., "Mild ultrasound-assisted synthesis of TiO 2 supported on magnetic nanocomposites for selective photo-oxidation of benzyl alcohol," Appl. Catal. B Environ., vol. 183, pp. 107-112, Apr. 2016, doi: 10.1016/j.apcatb.2015.10.034.

[42] M. Nasrollahzadeh, Z. Nezafat, M. G. Gorab, and M. Sajjadi, "Recent progresses in graphene-based (photo)catalysts for reduction of nitro compounds," Mol. Catal., vol. 484, no. December 2019, p. 110758, 2020, doi: 10.1016/j.mcat.2019.110758.

[43] M. Bordbar and N. Mortazavimanesh, "Green synthesis of Pd/walnut shell nanocomposite using Equisetum arvense L. leaf extract and its application for the reduction of 4-nitrophenol and organic dyes in a very short time," Environ. Sci. Pollut. Res., vol. 24, no. 4, pp. 4093-4104, 2017, doi: 10.1007/s11356016-8183-y.

[44] S. Saha, A. Pal, S. Kundu, S. Basu, and T. Pal, "Photochemical green synthesis of calcium-alginatestabilized ag and au nanoparticles and their catalytic application to 4-nitrophenol reduction," Langmuir, vol. 26, no. 4, pp. 2885-2893, 2010, doi: 10.1021/la902950x.

[45] M. Orlandi, D. Brenna, R. Harms, S. Jost, and M. Benaglia, "Recent Developments in the Reduction of Aromatic and Aliphatic Nitro Compounds to Amines," Org. Process Res. Dev., vol. 22, no. 4, pp. 430-445, 2018, doi: 10.1021/acs.oprd.6b00205.

[46] K. Zhang et al., "Facile synthesis of monodispersed Pd nanocatalysts decorated on graphene oxide for reduction of nitroaromatics in aqueous solution," Res. Chem. Intermed., vol. 45, no. 2, pp. 599-611, 2019, doi: 10.1007/s11164-018-3621-8.

[47] D. Formenti, F. Ferretti, F. K. Scharnagl, and M. Beller, "Reduction of Nitro Compounds Using 3d-NonNoble Metal Catalysts," Chem. Rev., vol. 119, no. 4, pp. 2611-2680, Feb. 2019, doi:

10.1021/acs.chemrev.8b00547.

[48] H. C. Brown and P. V. Ramachandran, "Sixty Years of Hydride Reductions," in ACS Symposium Series, vol. 641, 1996, pp. 1-30.

[49] L. Pasumansky, C. T. Goralski, and B. Singaram, "Lithium aminoborohydrides: Powerful, selective, airstable reducing agents," Org. Process Res. Dev., vol. 10, no. 5, pp. 959-970, 2006, doi:

10.1021/op0600759.

[50] S. Naghdi, M. Sajjadi, M. Nasrollahzadeh, K. Y. Rhee, S. M. Sajadi, and B. Jaleh, "Cuscuta reflexa leaf extract mediated green synthesis of the Cu nanoparticles on graphene oxide/manganese dioxide nanocomposite and its catalytic activity toward reduction of nitroarenes and organic dyes," J. Taiwan Inst. Chem. Eng., vol. 86, pp. 158-173, May 2018, doi: 10.1016/j.jtice.2017.12.017.

[51] K. M. Gayathri, S. Paramparambath, A. Satheesh, S. Selvam, and E. Kandasamy, "Reduction of aldehydes and ketones by NaBH4 in presence of 1-alkyl-1,2,4-triazolium salts," Mater. Today Proc., vol. 33, 
no. xxxx, pp. 2381-2384, 2020, doi: 10.1016/j.matpr.2020.05.470.

[52] H. Hu, J. H. Xin, H. Hu, X. Wang, D. Miao, and Y. Liu, "Synthesis and stabilization of metal nanocatalysts for reduction reactions - A review," J. Mater. Chem. A, vol. 3, no. 21, pp. 11157-11182, 2015, doi: 10.1039/c5ta00753d.

[53] B. Zeynizadeh and F. Shirini, "Mild and Efficient Method for Reduction of Aldehydes and Ketones with NaBH 4 in the Presence of Dowex1-x8," J. Chem. Res., vol. 2003, no. 6, pp. 335-339, Jun. 2003, doi: $10.3184 / 030823403103174290$.

[54] F. A. Westerhaus et al., "Heterogenized cobalt oxide catalysts for nitroarene reduction by pyrolysis of molecularly defined complexes," Nat. Chem., vol. 5, no. 6, pp. 537-543, 2013, doi: 10.1038/nchem.1645.

[55] M. Atarod, M. Nasrollahzadeh, and S. Mohammad Sajadi, "Euphorbia heterophylla leaf extract mediated green synthesis of $\mathrm{Ag} / \mathrm{TiO} 2$ nanocomposite and investigation of its excellent catalytic activity for reduction of variety of dyes in water," J. Colloid Interface Sci., vol. 462, pp. 272-279, Jan. 2016, doi: 10.1016/j.jcis.2015.09.073.

[56] T. Jiao et al., "Reduced Graphene Oxide-Based Silver Nanoparticle-Containing Composite Hydrogel as Highly Efficient Dye Catalysts for Wastewater Treatment," Sci. Rep., vol. 5, no. 1, p. 11873, Dec. 2015, doi: 10.1038/srep11873.

[57] A. Maleki, Z. Hajizadeh, and R. Firouzi-Haji, "Eco-friendly functionalization of magnetic halloysite nanotube with $\mathrm{SO} H \mathrm{H}$ for synthesis of dihydropyrimidinones," Microporous Mesoporous Mater., vol. 259, pp. 46-53, Mar. 2018, doi: 10.1016/j.micromeso.2017.09.034.

[58] Z. Varzi and A. Maleki, "Design and preparation of ZnS-ZnFe204: a green and efficient hybrid nanocatalyst for the multicomponent synthesis of 2,4,5-triaryl-1H-imidazoles," Appl. Organomet. Chem., vol. 33, no. 8, pp. 1-11, 2019, doi: 10.1002/aoc.5008.

[59] N. Vahedi-Notash, M. M. Heravi, A. Alhampour, and P. Mohammadi, "Ag nanoparticles immobilized on new mesoporous triazine-based carbon (MTC) as green and recoverable catalyst for reduction of nitroaromatic in aqueous media," Sci. Rep., vol. 10, no. 1, pp. 1-9, 2020, doi: 10.1038/s41598-020-742324.

\section{Tables}

Table 1. The effect of Biochar/ $/ \mathrm{Fe}_{3} \mathrm{O}_{4}$-Ag nanocatalyst amount, Temperature, Solvent, and $\mathrm{NaBH}_{4}{ }^{\mathrm{a}}$ 


\begin{tabular}{|c|c|c|c|c|c|c|}
\hline Entry & $\begin{array}{c}\text { Catalyst } \\
\text { (mg) }\end{array}$ & $\mathrm{T}\left({ }^{\circ} \mathrm{C}\right)$ & Solvent & $\begin{array}{l}\mathrm{NaBH}_{4} \\
(\mathrm{mmol})\end{array}$ & $\begin{array}{l}\text { Time } \\
(\mathrm{min})\end{array}$ & $\begin{array}{l}\text { Yield } \\
(\%)^{\mathrm{b}}\end{array}$ \\
\hline 1 & - & 25 & $\mathrm{H}_{2} \mathrm{O}$ & 1 & 40 & 97 \\
\hline 2 & 2 & 25 & $\mathrm{H}_{2} \mathrm{O}$ & 1 & 12 & 97 \\
\hline 3 & 3 & 25 & $\mathrm{H}_{2} \mathrm{O}$ & 1 & 10 & 97 \\
\hline 4 & 5 & 25 & $\mathrm{H}_{2} \mathrm{O}$ & 1 & 3 & 98 \\
\hline 5 & 5 & 50 & $\mathrm{H}_{2} \mathrm{O}$ & 1 & 2 & 98 \\
\hline 6 & 5 & 25 & Ethanol & 1 & 13 & 60 \\
\hline 7 & 5 & 25 & $\mathrm{H} 2 \mathrm{O}:$ Ethanol (1:1) & 1 & 5 & 85 \\
\hline 8 & 5 & 25 & Acetonitrile & 1 & 6 & 87 \\
\hline 9 & 5 & 25 & THF & 1 & 8 & 96 \\
\hline 10 & 5 & 25 & DMSO & 1 & 6 & 45 \\
\hline 11 & 5 & 25 & DMF & 1 & 9 & 75 \\
\hline 12 & 5 & 25 & $\mathrm{CHCl}_{3}$ & 1 & 30 & trace \\
\hline 13 & 5 & 25 & $\mathrm{H}_{2} \mathrm{O}$ & 0.5 & 10 & 95 \\
\hline 14 & 5 & 25 & $\mathrm{H}_{2} \mathrm{O}$ & 2 & 1 & 98 \\
\hline
\end{tabular}

a Reaction Condition: Benzaldehyde ( $0.5 \mathrm{mmol})$, Solvent $(2 \mathrm{~mL})$

b Isolated yield

Table 2. The reduction reaction of aldehyde and ketone compounds in the presence of biochar $/ \mathrm{Fe}_{3} \mathrm{O}_{4}-\mathrm{Ag}$ nanocatalyst ${ }^{\text {a }}$ 


\begin{tabular}{|c|c|c|c|c|}
\hline Entry & Aldehyde/Ketone & Product & Time (min) & Yield $(\%)^{b}$ \\
\hline 1 & & & 3 & 98 \\
\hline 2 & & & 4 & 98 \\
\hline 3 & & & 5 & 98 \\
\hline 4 & & & 7 & 97 \\
\hline 5 & & & 6 & 97 \\
\hline 6 & & & 10 & 95 \\
\hline 7 & & & 10 & 85 \\
\hline 8 & & & 10 & 90 \\
\hline 9 & & & 6 & 80 \\
\hline 10 & & & 45 & 60 \\
\hline 11 & & & 60 & 30 \\
\hline
\end{tabular}

${ }^{\text {a }}$ Reaction Condition: Aldehyde and Ketone compounds (0.5 mmol), Catalyst ( $\left.5 \mathrm{mg}\right), \mathrm{H}_{2} \mathrm{O}(2 \mathrm{~mL}), \mathrm{NaBH}_{4}$ (1 mmol), RT

b Isolated yield

Figures 


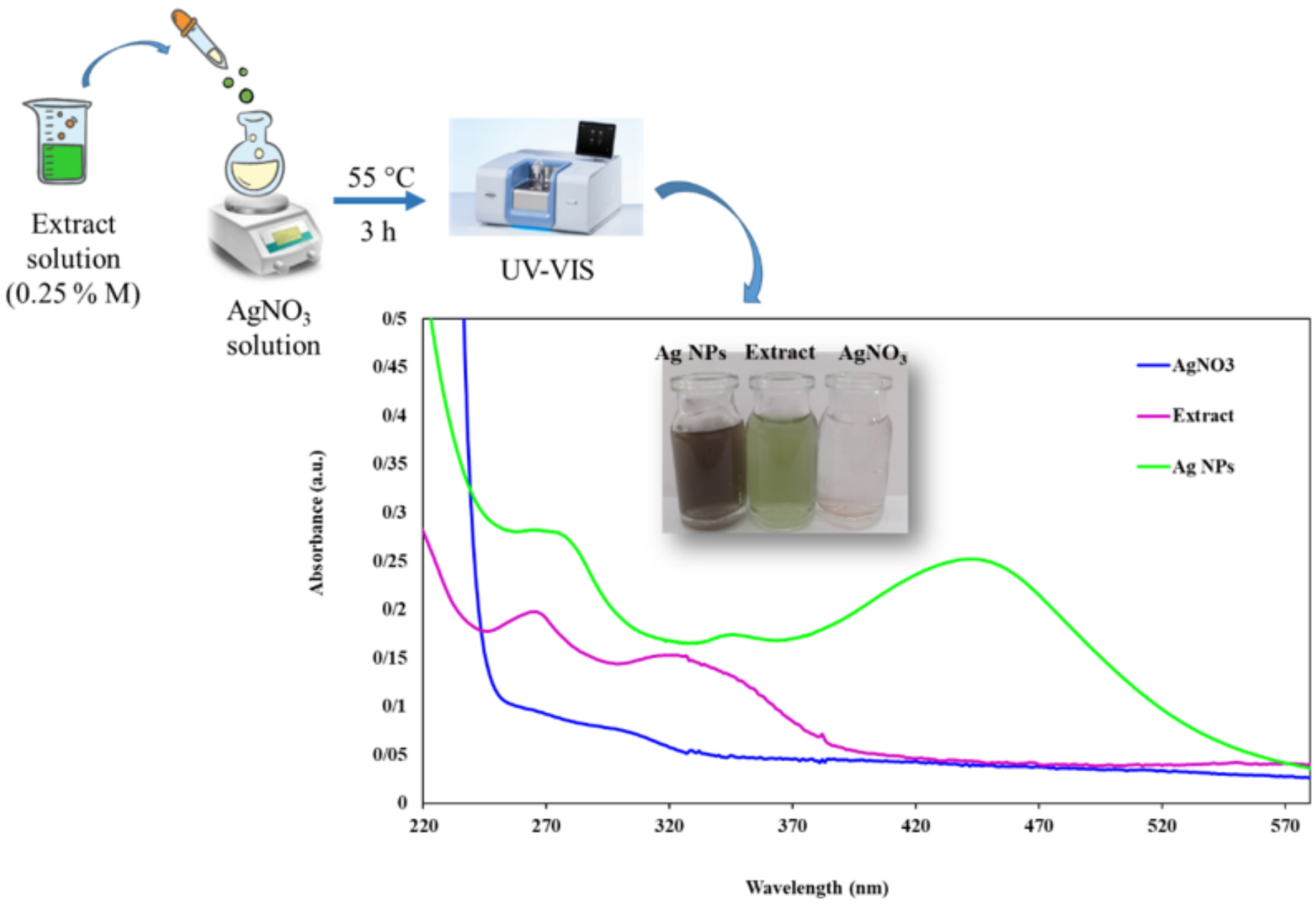

Figure 1

UV-Vis analysis of Celery leaf extract, pure AgNO3, and synthesized Ag NPs 


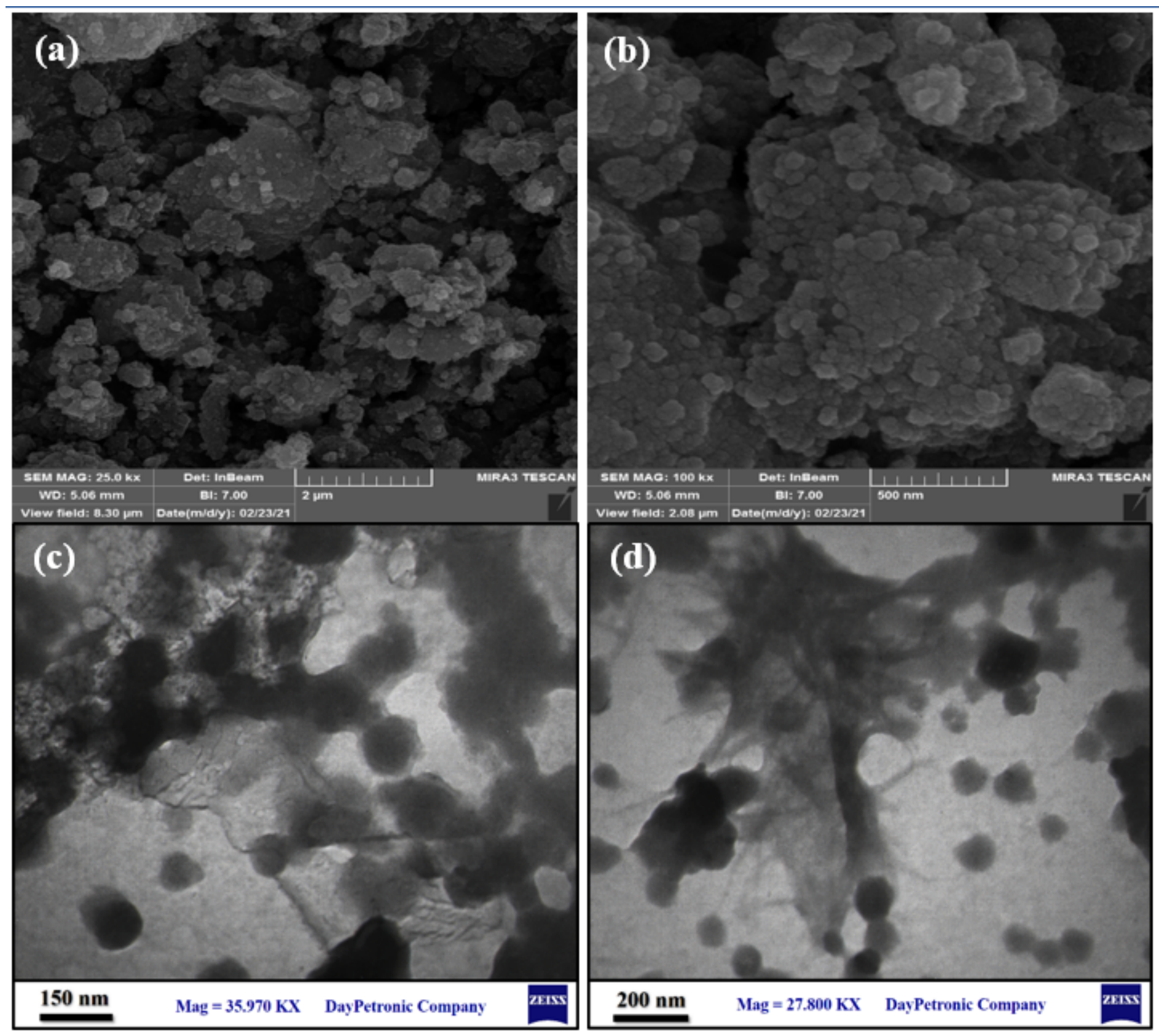

Figure 2

(a, b) FESEM images, and (c, d) TEM images of biochar/Fe304-Ag nanocomposite 

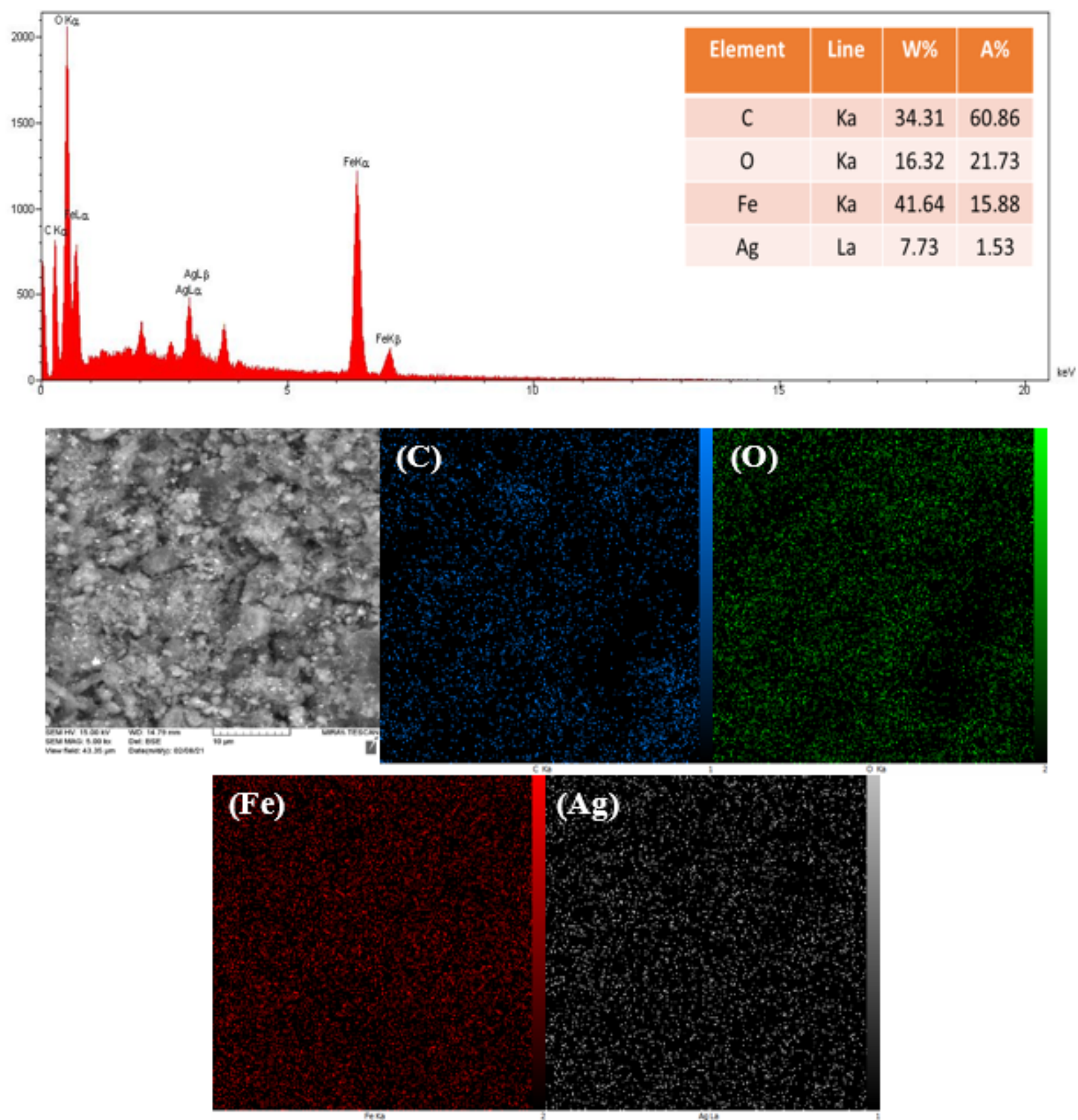

Figure 3

EDX-Mapping analysis of Biochar/Fe304-Ag nanocomposite 


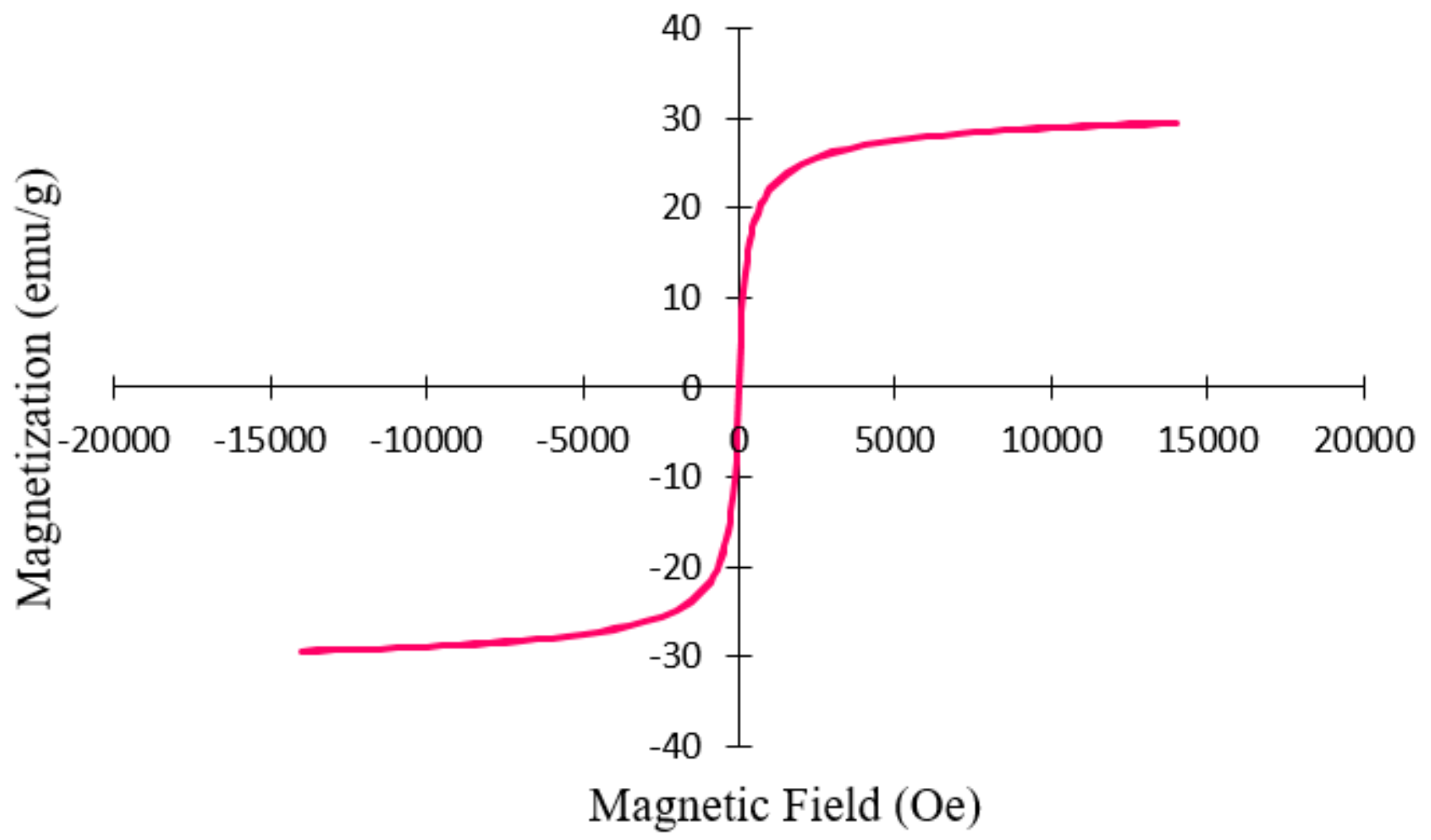

Figure 4

Magnetization curve of Biochar/Fe304-Ag nanocomposite

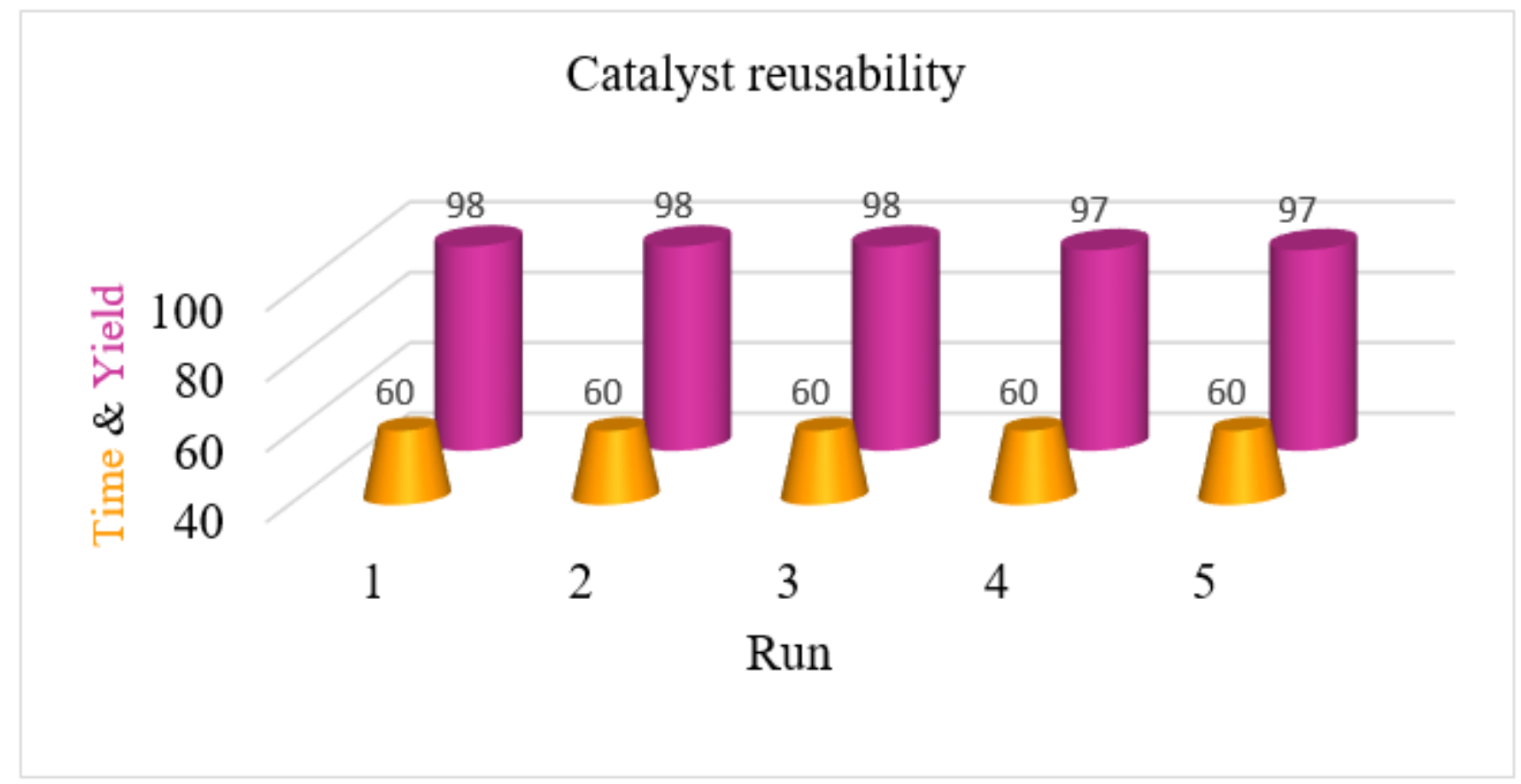

Figure 5

Reusability of the Biochar/Fe304-Ag nanocatalyst for the nitroaromatics reduction reaction 


\section{Catalyst reusibility}

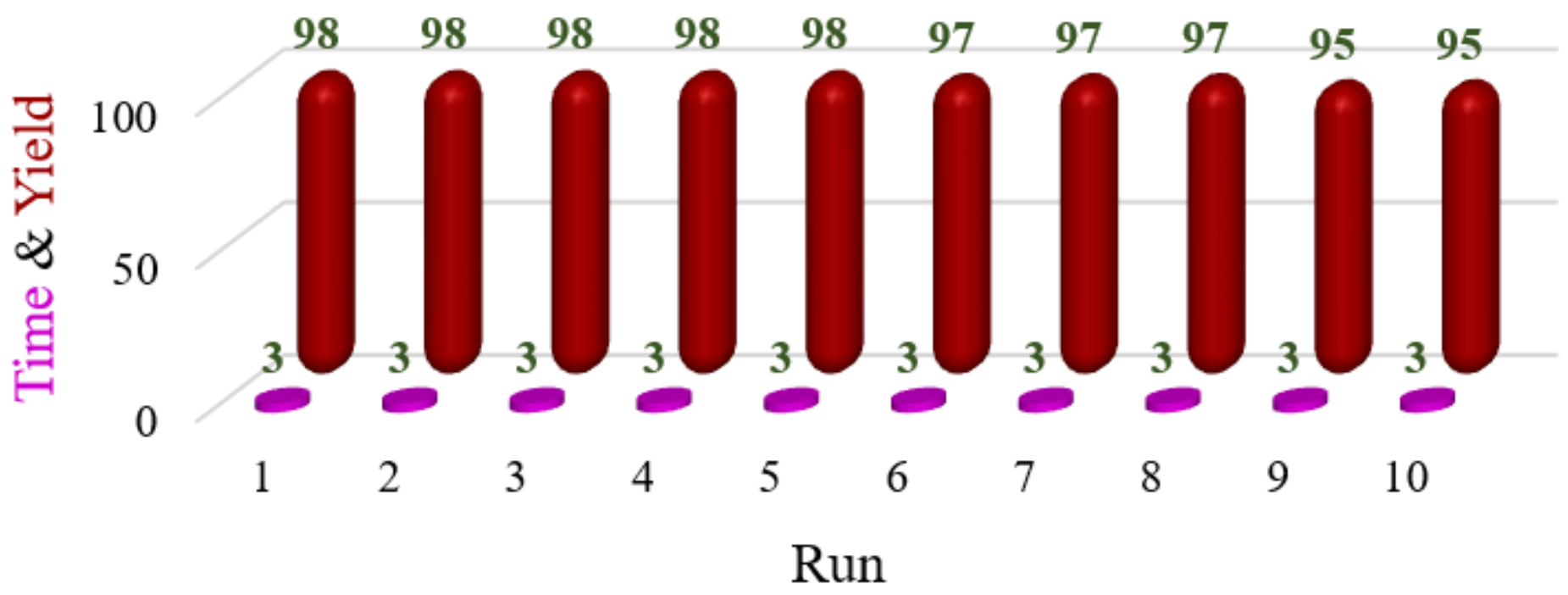

Figure 6

Reusability of the Biochar/Fe304-Ag nanocatalyst for Aldehydes and Ketones reduction reaction

\section{Supplementary Files}

This is a list of supplementary files associated with this preprint. Click to download.

- Supportinginformation.docx 